\title{
I verbi di servizio (modali, aspettuali e causativi) e i verbi supporto nelle grammatiche descrittive d'italiano dal 1953 al 2005
}

\section{Helping verbs (modal, aspectual, causative verbs) and light verbs in Italian descriptive grammars published between 1953 and 2005}

\author{
Anna Godzich \\ Università Adam Mickiewicz, Poznań \\ annas@amu.edu.pl
}

\begin{abstract}
The paper deals with Italian helping verbs (modal, aspectual, causative verbs) and light verbs which form complex predicates and with the rules that guide its use in Italian descriptive grammars published in Italy between 1953 and 2005. The author shows that those forms in both - traditional and contemporary Italian descriptive grammars are treated at the level of word classes whereas it could be more appropriate to discuss them as clause elements as they form complex predicates. In our opinion this way of describing such verbs is due to the tradition in Italy to focus on a form and not on function of an element. What is more, Italian grammarians tend to omit in Italian descriptive grammars noun predication and the role of semantic predicate ( $n$ pred.). The goal of the paper is to present the advantages of an integrated approach to helping verbs (modal, aspectual, causative verbs) and light verbs in modern Italian. The author emphasizes its importance for contemporary Italian FL syntax teaching.
\end{abstract}

Keywords: Italian FL syntax teaching, Italian helping verbs, auxiliary verbs, modal verbs, light verbs, complex predicates, Italian descriptive grammars

\section{PREMESSA}

Vorremmo cominciare la nostra riflessione sull'insegnamento della grammatica descrittiva della lingua italiana - sintassi della proposizione - agli studenti stranieri con un riferimento a "Dieci tesi per l'educazione linguistica democratica" formulate 
nell'ambito del Gruppo di Intervento e Studio nel Campo dell'Educazione Linguistica (G.I.S.C.E.L.) nell'ormai remoto 1975. Gli studiosi, trattando dell'inefficacia della pedagogia linguistica tradizionale, puntualizzavano la nocività di tale insegnamento grammaticale, poiché fondato «su teorie del funzionamento d'una lingua che sono antiquate (...)». Benché si trattasse dei discenti di madrelingua italiana, visto il metodo e gli strumenti d'insegnamento della grammatica della lingua italianasintassi agli studenti polacchi, riteniamo che la disapprovazione dei linguisti del G.I.S.C.E.L. possa essere applicabile pure all'insegnamento nostrano per motivi che in seguito elencheremo. Innanzitutto, la mancata inclusione nelle grammatiche descrittive italiane destinate ai discenti madrelingua di riferimenti a verbi pronominali o predicati nominali in senso largo (quindi quelli verbo-nominali). Pensiamo qua non solo a quelli segnalati da grammatiche tradizionali, ovvero a:

a) predicati nominali formati da essere, parere, sembrare, apparire, divenire, diventare + nome del predicato / complemento predicativo del soggetto,

b) verbi nascere, morire, vivere, crescere, rimanere, restare, risultare, farsi usati come verbi copulativi,

c) verbi appellativi come chiamare, dire, soprannominare usati al passivo,

d) verbi elettivi eleggere, nominare, proclamare, dichiarare usati al passivo,

e) verbi estimativi come considerare, stimare, reputare, ritenere, giudicare usati al passivo,

f) verbi effettivi fare, rendere, creare usati al passivo,

ma anche a tutti gli altri predicati (verbo-)nominali a struttura verbo supporto + nome predicativo (effettuare un'analisi, fare un salto che corrispondono ai verbi predicativi: analizzare, saltare) che non vengono segnalati quando si parla del concetto di proposizione. In conseguenza lo studente, non avendo ben chiara la tipologia e diversità di predicati, non riesce a svolgere gli esercizi in maniera corretta. Se non includiamo nell'elenco di predicati particolari quelli sopraddetti, in risultato allo studente mancheranno gli strumenti di analisi. È anche vero che si auspichi una maggiore semplicità di concetti che si introducono durante le lezioni, ma a parer nostro ciò non dovrebbe significare una semplificazione eccessiva.

Come possiamo osservare, nei manuali di grammatica italiana destinati ai discenti madrelingua con il termine di predicato nominale si definiscono i predicati composti da una classe di verbi copulativi piuttosto chiusa, di solito vengono elencati i predicati nominali canonici (è di nuovo il verbo ad essere l'elemento centrale della proposizione, mentre a possedere le proprietà di formarla sono anche altri elementi, come nome ${ }^{1}$, aggettivo od avverbio).

Visto che a dare difficoltà allo studente polacco durante le prime lezioni di sintassi della proposizione in italiano sono i predicati composti da verbi di servizio, si è deciso di dedicare il contributo proprio a quest'argomento.

\footnotetext{
${ }^{1}$ Vetulani (2012, p. 15) parla di «cechy zdaniotwórcze».
} 


\section{VERBI DI SERVIZIO}

Saper distinguere tra una proposizione con un predicato complesso e due proposizioni: una reggente ed una subordinata, risulta fondamentale per l'insegnamento della sintassi dell'italiano LS. Per facilitarne la distinzione agli studenti polacchi l'oggetto del presente studio sono i verbi di servizio, ossia quelli che svolgono una funzione vicaria nei confronti di altri verbi, da essi accompagnati. Si hanno in mente in questa sede «tutti i verbi [tranne gli ausiliari propriamente detti - nota dell'autrice] che si uniscono ad altri verbi per precisarne o arricchirne il significato» (Sensini, 2005, p. 242). Questi verbi «costituiscono con i verbi a cui si uniscono un'unità sintattica compatta e quindi formano un unico predicato» (Sensini, 2005, p. 242). La denominazione di verbi di servizio proposta dall'autore suddetto è ovviamente un iperonimo e all'interno del gruppo stesso possiamo distinguere i seguenti tipi di verbi, a seconda della funzione da essi svolta:

1.i verbi modali ( $\mathrm{o}$, in senso largo, servili): dovere, potere, volere ma anche osare, preferire, sapere (= essere in grado di), cercare di, tentare di, desiderare e simili;

2.i verbi aspettuali come accingersi $a$, mettersi a, cominciare $a$, incominciare $a$, continuare a, finire per / di, smettere di, solere (= essere soliti), ovverosia quelli che possono accompagnare altri verbi di modo indefinito precisando l'aspetto dell'azione (Sensini, 2005, p. 242); lo studioso puntualizza che nel caso dei suddetti verbi di modo indefinito si tratterà di «un infinito introdotto da una preposizione o un gerundio», tuttavia si tenga presente che può anche essere un infinito introdotto senza il tramite di una preposizione, p. es. In quel periodo solevo rincasare alle 21 ;

3.i verbi causativi (detti anche fattivi), cioè quelli «che si accompagnano a un altro verbo, posto all'infinito, per esprimere un'azione causata, cioè fatta eseguire o lasciata eseguire, dal soggetto» (Sensini, 2005, p. 242); sono verbi fare e lasciare (Ho fatto studiare gli studenti. Abbiamo lasciato giocare i bambini).

Si noti che non di rado tutti e tre i tipi di verbi funzionano nelle grammatiche descrittive ed enciclopedie della lingua italiana con il nome generico di verbi fraseologici (così per es. in Sensini, 2005, p. 242 o Jansen, 2010) ${ }^{2}$.

I verbi modali, aspettuali e causativi formano le cosiddette strutture perifrastiche (Cerruti, 2011) ${ }^{3}$ che i possono illustrare come segue:

$\mathrm{V}$ modale/aspettuale/causativo + (prep) + V nucleare:

Comincio / Mi metto / Mi accingo a studiare.

Finisco / Smetto / Mi sforzo / Cerco di studiare.

${ }^{2}$ Dato lo spazio concessoci per la presente riflessione, abbiamo tralasciato alcune questioni riguardanti l'argomento, ovverosia aspetto, lessicalizzazione e grammaticalizzazione.

${ }^{3}$ A proposito si veda anche Bertinetto (1996). 
Se non continuo a studiare, finisco per essere bocciato. Se non continuo a studiare, finisco con l'essere bocciato.

Sto per studiare.

Mi viene da piangere / ridere (in questo caso vigono certe restrizioni semantiche: *Mi viene da studiare / da partire).

Sto studiando.

Stando allo schema soprastante, nel caso di una struttura perifrastica, si tratterà quindi di un verbo modificatore «di significato relativamente leggero» (Cerruti, 2011) + verbo modificato (nucleare). Gli stessi verbi modificatori a loro volta si dividono in:

1. verbi lessicali (accingersi, avviarsi, cominciare, iniziare, continuare, proseguire, finire, smettere),

2. verbi grammaticali (fare, lasciare, stare, andare, venire) (Jansen, 2010).

Si può osservare che la suddetta distinzione si fa in base al grado di desemantizzazione di un dato verbo aspettuale: allorché i verbi lessicali indicano una fase dell'azione espressa dal verbo nucleare, quelli grammaticali sembrano più generici $\mathrm{e}$, in più, tendono a perdere il loro significato lessicale (Jansen, 2010).

Inoltre, come possiamo vedere, i verbi aspettuali e causativi non sono autonomi, svolgono la funzione di accompagnatori, come i verbi supporto nei predicati a struttura verbo supporto + nome predicativo (nella tradizione italiana detti predicati semantici) ${ }^{4}$.

A questo punto ci pare utile fare qualche cenno ai predicati complessi nelle grammatiche descrittive ed enciclopedie polacche, giusto per disporre di una prospettiva più ampia. In più, dalla nostra esperienza da docente risulta che sovente tale paragone incuriosisce i discenti. Notiamo allora che nelle grammatiche descrittive ed enciclopedie polacche si parla piuttosto di predicato complesso verbale (orzeczenie złożone werbalne), come per esempio il predicato introdotto da un verbo detto fasale (zaczynać, kończyć, kontynuować, przestać) (Polański, 1999, p. 98, Jagodziński ${ }^{5}$ ), modale (musieć, móc, potrafić, chcieć, zechcieć, raczyć), causativo (dawać, dać, kazać) ed altri, oppure di perifrasi verbale / predicato perifrastico (orzeczenie peryfrastyczne, peryfrastyczna konstrukcja analityczna, konstrukcja werbo-nominalna), composti da verbo supporto e nome predicativo: mieć nadzieję ('avere speranza', 'sperare'; non c'è in polacco *nadziejować), brać udziat ('prendere parte a', 'partecipare a'; non si usa *udziałować), mieć wptyw ('avere influsso su') (Polański, 1999, p. 431) ${ }^{6}$.

\footnotetext{
${ }^{4}$ Cfr. Gross (1981) e per l'italiano Elia, Vietri, Predicati semantici e Lessico-grammatica, http://infolingu.univ-mlv.fr/english/Bibliographie/Articles/predicatisemantici4.pdf (26.05.2019).

${ }^{5} \mathrm{http}: / /$ grzegorj.ugu.pl/gram/pl/skladnia05.html\#orzwerb (25.05.2019)

${ }^{6}$ Accanto alla perifrasi verbale / predicato perifrastico si hanno anche nelle lingue analitiche le cosiddette forme perifrastiche di parole, dette anche forme analitiche o forme descrittive. Ambedue, ovvero sia le forme analitiche, sia i predicati perifrastici sono esempi di perifrasi grammaticale (ibidem).
} 
Notiamo ancora che nella tradizione grammaticale polacca i verbi modificatori nei costrutti verbali del tipo aller + inf., venir de + inf. vengono definiti ausiliari (ivi, p. 98) ${ }^{7}$. La funzione degli ausiliari può essere quella di esprimere un valore modale o temporale. Vediamo quindi che l'approccio degli studiosi polacchi risulta più generico di quello degli italiani dal momento che tendono a ragruppare i verbi dai valori particolari nello stesso insieme di verbi detti ausiliari. Viene quindi messa in rilievo la loro funzione vicaria nei confronti del verbo nucleare / modificato e non tanto la loro semantica.

Visto e considerato quanto sopra, il nostro contributo verterà sui verbi modali, aspettuali e causativi di solito omessi o appena segnalati nei manuali d'italiano degli anni Sessanta e Settanta del Novecento destinati al discente italiano e in quelli più recenti trattati ma non in maniera esaustiva.

Disponendo dello spazio sì limitato, non possiamo discutere in questa sede la nozione del predicato, tuttavia va precisato che per i bisogni della nostra ricerca come tale si intende il nucleo informativo della proposizione (Radimský, 2009, p. 15). «La funzione del predicato può essere svolta da verbi autonomi di forma finita, semanticamente pieni» (Polański, 1999, p. 410; traduzione dell'autrice). «Nel caso di elementi che non possono da soli svolgere la funzione del predicato, vengono considerati predicati i costrutti formati da un verbo ausiliare (sinsematico) + elementi suddetti (di solito si tratta di un aggettivo o nome, più raramente un avverbio)» (idem, traduzione dell'autrice). Notiamo che neanche i verbi modali, aspettuali e causativi sono autonomi.

Sui verbi modali converrebbe ancora aggiungere che dal punto di vista formale essi reggono complementi oggetto del verbo al quale fanno le veci del verbo di servizio (Serianni, Della Valle, Patota, 2003, p. 221):

Voglio (che cosa?) mangiare una mela.

Formalmente si ha qui un infinito con valore nominale che, stando ai suddetti studiosi, può essere un complemento oggetto dal momento che ha lo stesso valore del sostantivo. In più, in Grammatica Italiana Treccani on-line leggiamo che «le proposizioni oggettive sono proposizioni $\rightarrow$ completive che svolgono, per così dire, la funzione del complemento $\rightarrow$ oggettor ${ }^{8}$.

A questo punto occorrerebbe corredare l'approccio formale di quello semantico: mentre nel caso di complementi oggetto propriamente detti il verbo reggenteli risulta di solito semanticamente pieno:

\footnotetext{
Un esempio di forme analitiche sono i tempi composti italiani, inglesi o francesi, oppure in polacco il futuro dei verbi imperfettivi: będę pisal, będziesz ogladal (ivi, p. 432).

${ }^{7}$ Ciò concerne anche i verbi modali e temporali: devoir, pouvoir, can, shall, will (ibidem).

${ }^{8} \mathrm{http}: / /$ www.treccani.it/enciclopedia/proposizioni-oggettive_(La-grammatica-italiana)/ (26.05.2019). Tuttavia, al contempo secondo l'Enciclopedia dell'Italiano Treccani, il verbo modale fa blocco sintattico con il verbo da esso retto: Devo passare per il bosco / per andare dalla nonna $=>$ il verbo modale dovere fa blocco sintattico con passare, http://www.treccani.it/lingua italiana/domande _e_risposte/grammatica/grammatica_780.html (27.05.2019).
} 
Cerco (che cosa?) la mia agenda.

Vedo (che cosa?) gli uccelli.

Quando si hanno i nomi predicativi del tipo analisi o salto nelle proposizioni:

Effettuiamo (che cosa?) un'analisi.

o Facciamo (che cosa?) un salto.

Si può notare che i verbi (supporto) in ambedue $\mathrm{i}$ casi non sono semanticamente pieni e hanno gli equivalenti sintetici (analizzare, saltare). Cerco, vado designano un'azione ben precisa, mentre effettuare e fare non lo fanno: semanticamente restano incompleti, bisognosi di un elemento che li renda pieni. Effettuare restringe solo il numero di sostantivi che ci possono essere collocati ${ }^{9}$.

Entrambi i fenomeni pertanto (sia i verbi modali, aspettuali e causativi che i verbi supporto) a nostro parere rientrerebbero nel quadro didattico della proposizione. Cionondimeno, i grammatici italiani paiono restii a trattare i predicati (verbo-)nominali nelle grammatiche descrittive.

\section{IL CORPUS}

Come corpus della presente ricerca abbiamo scelto alcuni manuali di grammatica tradizionali (tutti per le scuole medie (superiori)) considerati da noi rappresentativi per il periodo in cui sono stati pubblicati (a cominciare dagli anni Cinquanta del Novecento fino al 2005). Si tratterà quindi di un approccio diacronico. I manuali analizzati vengono elencati in seguito in ordine cronologico:

1. Paniate E. (1953), Analisi logica per la scuola media, Torino.

2. Schiannini D., Guardigli P.L., Bastianello F., Castellini P. (1974 [1969]), Il libro Garzanti della lingua italiana per le scuole medie superiori, Milano.

3. Panozzo U., Sacerdoti N. (1979), Dalla parola al linguaggio. Strutture, origini, evoluzione della lingua italiana per la scuola media, Firenze.

4. Prandi M. (1991), Grammatica della lingua italiana per le scuole medie superiori, Torino.

5. Serianni L., Della Valle V., Patota G. (2003), L'italiano parlato e scritto. Grammatica di riferimento e manuale delle abilità testuali con Schede di autoverifica, Milano.

6. Sensini M. (2005), La lingua e i testi. La riflessione sulla lingua, Milano.

\footnotetext{
${ }^{9} \mathrm{Si}$ veda a proposito la tipologia proposta da Ježek (2004, p. 193): la studiosa propone una distinzione tra i predicati con verbo supporto neutro (avere l'influenza, fare una discussione, avere caldo) e quelli con verbo supporto marcato (covare l'influenza, intavolare una discussione, scoppiare di caldo). Si nota subito che dal verbo supporto marcato si possono ricavare informazioni riguardanti la fase di svolgimento dell'azione (intavolare una discussione) nonché quelle sulla quantità (scoppiare di caldo che indica l'intensità).
} 
Inoltre, la ricerca è stata corredata di seguenti grammatiche di consultazione ed Enciclopedia Treccani:

1. Serianni L. (1988), Il verbo, [in:] Grammatica italiana: italiano comune e lingua letteraria: suoni, forme, costrutti, a c. di L. Serianni con la collaborazione di A. Castelvecchi, Torino, pp. 321-409.

2. Bertinetto P.M. (1996), Le perifrasi verbali, [in:] Grande grammatica italiana di consultazione. Vol. 2: I sintagmi verbale, aggettivale, avverbiale. La subordinazione a c. di P. M. Bertinetto, Bologna, pp. 129-161.

3. Jansen H. (2010), Verbi fraseologici, [in:] Enciclopedia dell'Italiano Treccani ${ }^{10}$.

4. Cerruti M. (2011), Strutture perifrastiche, [in:] Enciclopedia dell'Italiano Treccani ${ }^{11}$.

5. Serianni L. (2015 [1997]), Sintassi del periodo, [in:] Italiano. Grammatica italiana. Ortografia e morfologia. Analisi logica e grammaticale. Sintassi della proposizione e del periodo a c. di L. Serianni con la collaborazione di A. Castelvecchi. Glossario di G. Patota, Milano, pp. 368-440.

6. Cimaglia R., Verbi modali, [in:] Enciclopedia dell'Italiano Treccani ${ }^{12}$.

A nostro avviso con questo corpus si può verificare qual è il posto dato dai grammatici italiani all'argomento in questione e se le informazioni presenti nei manuali italiani vadano ulteriormente corredate di altri materiali (non di rado d'autore) durante le lezioni di sintassi d'italiano ad un corso accademico tenuto fuori Penisola.

\section{L'ANALISI}

Il primo aspetto che abbiamo deciso di appurare era quello riguardante la denominazione del fenomeno cui dedichiamo la nostra breve riflessione linguistica, ovvero con quale nome funzionano nelle grammatiche tradizionali d'italiano i verbi modali, aspettuali e causativi. Dall'accurato esame risulta che gli studiosi italiani appaiono abbastanza unanimi nel designarli come verbi servili e fraseologici:

Tab. 1. I verbi in questione nelle grammatiche descrittive della lingua italiana

\begin{tabular}{|l|l|l|l|l|l|l|}
\hline & \multicolumn{1}{|c|}{ Paniate (1953) } & $\begin{array}{l}\text { Libro Garzanti della } \\
\text { lingua italiana (1974 } \\
[1969])\end{array}$ & $\begin{array}{l}\text { Panozzo, } \\
\text { Sacerdoti } \\
\text { (1979) }\end{array}$ & Prandi (1991) & $\begin{array}{l}\text { Serianni, Della } \\
\text { Valle, Patota } \\
\text { (2003) }\end{array}$ & \multicolumn{1}{c|}{ Sensini (2005) } \\
\hline $\begin{array}{l}\text { Nome sotto } \\
\text { cui appaiono } \\
\text { i verbi modali, } \\
\text { aspettuali } \\
\text { e causativi }\end{array}$ & $\begin{array}{l}\text { verbi fraseologici (in- } \\
\text { tesi come verbi aspet- } \\
\text { tuali) e verbi servili } \\
\text { (intesi come verbi } \\
\text { modali propriamente } \\
\text { detti e altri verbi ser- } \\
\text { vili come desiderare) }\end{array}$ & $\begin{array}{l}\text { verbi servili (proba- } \\
\text { bilmente intesi come } \\
\text { verbi modali) e verbi } \\
\text { fraseologici (intesi } \\
\text { come i verbi aspet- } \\
\text { tuali t verbo sapere } \\
\text { e desiderare) }\end{array}$ & $\begin{array}{l}\text { verbi servili } \\
\text { (intesi come } \\
\text { verbi servili, } \\
\text { aspettuali } \\
\text { e causativi) }\end{array}$ & $\begin{array}{l}\text { verbi servili, verbi } \\
\text { fraseologici (intesi } \\
\text { come verbi aspettuali) } \\
\text { tra cui vengono } \\
\text { annoverate anche } \\
\text { locuzioni predicative } \\
\text { (essere sul punto di) }\end{array}$ & $\begin{array}{l}\text { verbi servili } \\
\text { (intesi come } \\
\text { modali), fras- } \\
\text { eologici (intesi } \\
\text { come aspet- } \\
\text { tuali), causa- } \\
\text { tivi }\end{array}$ & $\begin{array}{l}\text { verbi fraseologici } \\
\text { (inclusi i verbi } \\
\text { servili, aspettuali } \\
\text { e causativi) }\end{array}$ \\
\hline
\end{tabular}

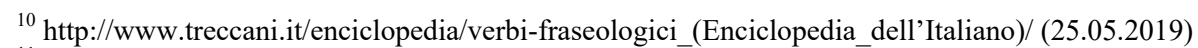

${ }^{11} \mathrm{http} / / / \mathrm{www}$. treccani.it/enciclopedia/strutture-perifrastiche_(Enciclopedia_dell'Italiano)/ (25.05.2019)

$12 \mathrm{http} / / /$ www.treccani.it/enciclopedia/verbi-modali_(Enciclopedia-dell'Italiano)/ (25.05.2019)
} 
Avendo il nostro studio un approccio diacronico, abbiamo inoltre analizzato i verbi modali, aspettuali e causativi nelle grammatiche - corpus della presente trattazione a seconda del posto del manuale in cui vengono trattati dagli autori (ovvero se messi nelle parti del discorso o in quanto elementi della proposizione). In base a ciò si possono fornire $i$ dati seguenti:

Tab. 2. La presenza dettagliata dei verbi modali, aspettuali e causativi nelle grammatiche descrittive italiane tradizionali ed attuali

\begin{tabular}{|c|c|c|c|c|c|c|}
\hline & $\begin{array}{l}\text { Paniate } \\
(1953)\end{array}$ & $\begin{array}{c}\text { Libro Garzanti della } \\
\text { lingua italiana } \\
(1974[1969])\end{array}$ & $\begin{array}{c}\text { Panozzo, Sacerdoti } \\
\text { (1979) }\end{array}$ & $\begin{array}{l}\text { Prandi } \\
(1991)\end{array}$ & $\begin{array}{c}\text { Serianni, Della } \\
\text { Valle, Patota } \\
(2003)\end{array}$ & $\begin{array}{l}\text { Sensini } \\
(2005)\end{array}$ \\
\hline verbi predicativi & + & + & + & + & + & + \\
\hline verbi copulativi & + & + & + & + & + & + \\
\hline verbi aspettuali & + & + & + & + & + & + \\
\hline verbi modali / servili & + & + & + & + & + & + \\
\hline $\begin{array}{l}\text { verbi supporto + nomi } \\
\text { / aggettivi / avverbi } \\
\text { predicativi }\end{array}$ & $\begin{array}{l}\text { non } \\
\text { concerne }\end{array}$ & - & $\begin{array}{l}\text { - (i predicati avere } \\
\text { intenzione di ed } \\
\text { essere sul punto di } \\
\text { annoverati tra i ver- } \\
\text { bi servili) }\end{array}$ & - & - & - \\
\hline $\begin{array}{l}\text { esercizi pratici verbi } \\
\text { predicativi vs. quelli } \\
\text { copulativi }\end{array}$ & - & + & + & + & + & + \\
\hline $\begin{array}{l}\text { esercizi pratici verbi } \\
\text { aspettuali }\end{array}$ & - & + & + & + & + & + \\
\hline $\begin{array}{l}\text { esercizi pratici verbi } \\
\text { ausiliari propriamente } \\
\text { detti }\end{array}$ & - & + & + & + & + & + \\
\hline $\begin{array}{l}\text { esercizi pratici verbi } \\
\text { causativi }\end{array}$ & - & - & - & - & + & + \\
\hline $\begin{array}{l}\text { esercizi pratici verbi } \\
\text { modali / servili }\end{array}$ & - & + & $\begin{array}{l}+ \text { (in genere in tutti } \\
\text { gli esercizi del ma- } \\
\text { nuale si tratta di tro- } \\
\text { vare il predicato } \\
\text { e distinguere altri } \\
\text { elementi della pro- } \\
\text { posizione) }\end{array}$ & + & + & + \\
\hline $\begin{array}{l}\text { verbi modali, aspet- } \\
\text { tuali e causativi trattati } \\
\text { nei capitoli dedicati } \\
\text { alle parti del discorso }\end{array}$ & - & - & + & + & + & $\begin{array}{l}+ \text { (con un'informa- } \\
\text { zione da parte } \\
\text { dell'autore: «in vista } \\
\text { dell'analisi logica» } \\
\text { [p. 242]) }\end{array}$ \\
\hline $\begin{array}{l}\text { verbi modali, aspet- } \\
\text { tuali e causativi trattati } \\
\text { nei capitoli dedicati } \\
\text { alla proposizione }\end{array}$ & - & + & + & - & - & + \\
\hline
\end{tabular}


Dalla tabella soprastante si evince che a prevalere nelle grammatiche tradizionali italiane è l'approccio che vuole studiare $i$ verbi in questione nella parte dedicata al verbo, per cui viene messa in rilievo la funzione grammaticale, la parte del discorso («verbi modali, aspettuali e causativi nelle parti del discorso»), mentre per quanto riguarda la funzione logica all'interno della proposizione i verbi modali, aspettuali e causativi non vengono più trattati. $\mathrm{Ci}$ si nota anche la mancanza di esercizi miranti ad individuare un tipo sì particolare di verbi che sono i verbi oggetto del contributo. In più, viste e considerate le informazioni contenute nella Tab. 2, possiamo costatare che nelle grammatiche descrittive italiane si suole distinguere il predicato verbale da quello nominale (tradizionale, canonico), ci sono anche esercizi riguardanti questi argomenti. È così sia in Paniate sia in tutti gli altri manuali analizzati, mentre ai verbi modali, aspettuali e causativi si accenna appena nella prima grammatica esaminata, quella del 1953 (Paniate, 1953, p. 131).

\section{CONSIDERAZIONI FINALI}

Avendo svolto l'analisi, possiamo trarre le conclusioni seguenti:

1. In genere $i$ verbi in questione vengono trattati nei capitoli dedicati alle parti del discorso. Si osserva pertanto un forte legame col passato e la tradizione grammaticale in Italia che si manifesta nella prevalenza della forma sulla semantica e funzione.

2. Nelle grammatiche attuali (Serianni, Della Valle, Patota (2003), Sensini [2005]) si nota un approccio più dettagliato ed accurato ai verbi modali, aspettuali e causativi che nelle grammatiche tradizionali, quelle degli anni Sessanta e Settanta del secolo precedente: in effetti essi vengono introdotti a seconda della categoria: a cominciare da quelli modali / servili, aspettuali fino ai verbi causativi. Così per esempio nelle due grammatiche più recenti, ovvero in quella di Serianni, Della Valle, Patota (2003) nonché in Sensini (2005).

3. Alcune tipologie proposte dai grammatici si intersecano (p. es. i verbi fraseologici intesi solamente come quelli aspettuali (in Jansen, 2010) vs. i verbi fraseologici considerati come i verbi modali, aspettuali e causativi, fuorché gli ausiliari (in Sensini, 2005, p. 242) o verbi modali in senso stretto (ovvero dovere, potere, volere giacché solo essi ammettono un pronome atono sia in posizione proclitica che in quella enclitica) ed in senso largo (inclusi anche gli altri verbi modali o servili come osare, preferire, desiderare ecc. [cfr. Ci$\left.\left.\operatorname{maglia}^{13}\right]\right)$.

\footnotetext{
${ }^{13}$ http://www.treccani.it/enciclopedia/verbi-modali_(Enciclopedia-dell'Italiano)/ (26.05.2019)
} 
4. Perciò forse sarebbe opportuno introdurre la nozione di predicato complesso che includa sia il predicato complesso verbale composto da un verbo modale / aspettuale / causativo + verbo nucleare, sia il predicato verbo-nominale a struttura verbo supporto + nome / aggettivo / avverbio predicativo, sia il tradizionale, canonico predicato nominale a struttura copula + nome del predicato che effettivaente viene trattato nei manuali analizzati.

5. Converrebbe pure preparare degli esercizi che mirino ad individuare un dato tipo di predicato, mentre si hanno quasi solamente esercizi generici che fanno trovare il predicato. 


\section{BIBLIOGRAFIA}

Opere di riferimento

Elia, A., Vietri, S. Predicati semantici e Lessico-grammatica. Retrieved from: http://infolingu. univ-mlv.fr/english/Bibliographie/Articles/predicatisemantici4.pdf.

Godzich, A. (2017). L'insegnamento dell'italiano dentro e fuori la Penisola - verbi fraseologici e servili nei manuali scelti di sintassi d'italiano. In A. Paleta, M. Wrana (eds.), L'Italia come specchio dell'Europa e l'Europa come specchio dell'Italia nei tempi antichi e moderni. Atti del VI Incontro dei Giovani Italianisti Polacchi (pp. 77-91). Kraków: Collegium Colmbinum.

Gross, M. (1981). Les bases empiriques de la notion de prédicat sémantique. Langages, 63, 7-53. doi.org/10.3406/lgge.1981.1875

Gruppo di Intervento e Studio nel Campo dell'Educazione Linguistica (G.I.S.C.E.L.), Dieci tesi per l'educazione linguistica democratica. Retrieved from: http://www.giscel.it/?q=content/diecitesi-leducazione-linguistica-democratica.

Ježek, E. (2004). Types et degrés de verbes supports en italien. Linguisticae Investigationes, 27 (2), 185-201.

Polański, K. (ed.) (1999). Encyklopedia językoznawstwa ogólnego. Wrocław: Zakład Narodowy im. Ossolińskich.

Radimský, J. (2009). Tempo, aspetto e diatesi nel predicato nominale. Études Romanes de Brno, $30(1), 15-26$.

Vetulani, G. (2012). Kolokacje werbo-nominalne jako samodzielne jednostki języka. Syntaktyczny stownik kolokacji werbo-nominalnych języka polskiego na potrzeby zastosowań informatycznych, cz. I. Poznań: Wydawnictwo Naukowe UAM.

Grammatiche descrittive

Jagodziński, G. Składnia języka polskiego. Sposoby wyrażania poszczególnych części zdania. Orzeczenie. Homepage. Retrieved May 25, 2019 from http://grzegorj.ugu.pl/gram/pl/gram00.html.

Paniate, E. (1953). Analisi logica per la scuola media. Torino: S. Lattes \& C. Editori.

Panozzo, U., Sacerdoti, N. (1979). Dalla parola al linguaggio. Strutture, origini, evoluzione della lingua italiana per la scuola media. Firenze: Le Monnier.

Prandi, M. (1991). Grammatica della lingua italiana per le scuole medie superiori. Torino: Petrini.

Schiannini, D., Guardigli, P.L., Bastianello, F., Castellini, P. (1974 [1969]). Il libro Garzanti della lingua italiana per le scuole medie superiori. Milano: Garzanti.

Sensini, M. (2005). La lingua e i testi. La riflessione sulla lingua. Milano: Arnoldo Mondadori Scuola.

Serianni, L., Della Valle, V., Patota, G. (2003). L'italiano parlato e scritto. Grammatica di riferimento e manuale delle abilità testuali con Schede di autoverifica. Milano: Mondadori.

Grammatiche di consultazione

Bertinetto, P.M. (1996). Le perifrasi verbali. In P.M. Bertinetto (ed.), Grande grammatica italiana di consultazione, vol. 2: I sintagmi verbale, aggettivale, avverbiale. La subordinazione (pp. 129-161). Bologna: Il Mulino.

Cerruti, M. (2011). Strutture perifrastiche. Retrieved from: Enciclopedia dell'Italiano Treccani, $\mathrm{http} / / / w w w . t r e c c a n i . i t / e n c i c l o p e d i a /$ strutture-perifrastiche_(Enciclopedia_dell'Italiano)/. 
Cimaglia, R. Verbi modali. Retrieved from: Enciclopedia dell'Italiano Treccani, http://www. treccani.it/enciclopedia/verbi-modali_(Enciclopedia-dell'Italiano)/.

Grammatica Italiana Treccani on-line, Le proposizioni oggettive. Retrieved from: http://www. treccani.it/enciclopedia/proposizioni-oggettive_(La-grammatica-italiana)/.

Jansen, H. (2010). Verbi fraseologici. Retrieved from: Enciclopedia dell'Italiano Treccani, http://www.treccani.it/enciclopedia/verbi-fraseologici_(Enciclopedia_dell'Italiano)/.

Serianni, L. (1988). Il verbo. In L. Serianni in collaboration with A. Castelvecchi, Grammatica italiana: italiano comune e lingua letteraria: suoni, forme, costrutti (pp. 321-409). Torino: UTET.

Serianni, L. (2015 [1997]). Sintassi del periodo. In L. Serianni in collaboration with A. Castelvecchi, glossary by G. Patota, Italiano. Grammatica italiana. Ortografia e morfologia. Analisi logica e grammaticale. Sintassi della proposizione e del periodo (pp. 368-440). Milano: Garzanti.

Treccani.it, sezione: „Domande e risposte”, http://www.treccani.it/lingua_italiana/domande_e_ risposte/grammatica/grammatica_780.html. 